\title{
Adecuación de los libros de texto al Marco Común Europeo de Referencia para las Lenguas (MCERL) ${ }^{1}$
}

\author{
ANTONIO R. RoldÁn TAPIA \\ Dolores Lucena SERrano \\ Laura Mesenger Coca \\ José E. RoldÁn Ruiz \\ Mónica Sánchez Carmona \\ Universidad de Córdoba
}

Recibido: 1 febrero 2008 / Aceptado: 11 junio 2008

ISSN: $1697-7467$

\begin{abstract}
RESUMEN: El Marco Común Europeo de Referencia para las Lenguas (MCERL) se ha convertido en un elemento articulador de las políticas de enseñanza, aprendizaje y evaluación de lenguas en el entorno europeo. En él, se describen los niveles de competencia que se estando utilizando para valorar el conocimiento que un usuario tiene de cualquier lengua. Este estudio analiza cuál es el nivel de adecuación a los niveles de competencia del MCERL y sus escalas de descriptores de tres libros de texto habitualmente utilizados en el primer curso de Educación Secundaria.

Palabras clave: MCERL, niveles de competencia, descriptores, libros de texto.
\end{abstract}

\section{Textbook Adaptation to Common European Framework for Languages}

\begin{abstract}
The Common European Framework of Reference (CEFR) has become the tool that gives sense to all the European policies that have to do with language teaching, learning and evaluation. The CEFR includes a detailed description of the levels of competence that show what a language user can do with the languages he knows or uses. This piece of research analyzes to what extent there exists a correlation between these levels of competence and their descriptors, and the content of three commonly used books for the first year of Secondary Education.
\end{abstract}

Key words: CEFR, competence levels, descriptors, textbooks.

\footnotetext{
${ }^{1}$ Resultado del Proyecto de Investigación Educativa (PIV 007-06), titulado «El Marco Común Europeo de Referencia en los libros de texto de inglés», y financiado por la Consejería de Educación de la Junta de Andalucía, según Resolución de 15 de diciembre de 2006 (BOJA de 9 de enero de 2007).
} 


\section{INTRODUCCIÓN}

En el año 2001, después de un largo pilotaje, la División de Política Lingüística del Consejo de Europa (Council of Europe, 2001a) sacaba a la luz un documento, cuya versión española conocemos como Marco Común Europeo de Referencia para las lenguas: aprendizaje, enseñanza y evaluación (de aquí en adelante, MCERL), que venía a sustituir al Nivel Umbral [Threshold Level], publicado en 1970 y convertido en referencia de primer orden en los primeros años de desarrollo del enfoque comunicativo. En relación a esta publicación, en la Resolución 1539 (2001) del Consejo de Europa (Council of Europe 2001b), relativa al «Año Europeo de las Lenguas», en el punto 11, apartado iii, se decía expresamente:

encourage the relevant institutions to use the Common European Framework of Reference drawn up by the Council of Europe to develop their language policies, so as to ensure the quality of language teaching and learning and improve international co-ordination;

[animar a las instituciones responsables a utilizar el Marco Común Europeo de Referencia elaborado por el Consejo de Europa para desarrollar sus políticas lingüísticas, a fin de garantizar la calidad de la enseñanza y el aprendizaje de lenguas y mejorar la coordinación internacional]

El Marco es un documento de consulta o referencia, cuyo objetivo es homogeneizar los niveles de aprendizaje, uso y evaluación de las lenguas, en el que el plurilingüismo es el concepto clave en torno al cual se estructura todo el contenido y en torno al cual se fijan los objetivos delimitados en él. El Marco no es un documento que revolucione la práctica docente pero que sí nos lleva a una reflexión sobre los mecanismos, criterios y procedimientos reenseñanza y aprendizaje de lenguas.

Se establecen seis niveles de competencia para describir el grado ascendente de competencia comunicativa del aprendiz: $A 1$ y $A 2$, propios del Usuario Básico; $B 1$ y B2, propios del Usuario Independiente, y $C 1$ y $C 2$, que representan al Usuario Competente. Los niveles de competencia están especificados de forma detallada con descriptores que hacen referencia a lo que el usuario es capaz de hacer en cada una de las destrezas; siendo importante que el progreso del aprendiz, de un nivel de competencia a otro, no tiene por qué ser año a año, ni curso a curso, pudiendo variar este progreso en función de las lenguas, el tiempo empleado en su aprendizaje, la edad de iniciación, las destrezas practicadas, etc.

Desde entonces, distintas iniciativas se han puesto en marcha por parte de las administraciones educativas nacionales y regionales: el desarrollo del Portfolio de Lenguas en los distintos niveles, el incremento de la enseñanza de lenguas, en general, en el sistema educativo, con diferentes formatos pedagógicos, el establecimiento de una red de centros bilingües, etc.

La administración educativa que nos compete, la Consejería de Educación de la Junta de Andalucía, aprobó en Consejo de Gobierno el Plan de Fomento de Plurilingüismo en Andalucía, según el Acuerdo de 22 de Marzo de 2005 (Consejería de Educación, 2005a). Desde entonces, se ha desarrollado un conjunto de iniciativas importantes (centros bilingües, cursos de formación para alumnado y profesorado, y desarrollo de materiales curriculares para las áreas no-lingüísticas impartidas en segunda lengua, entre otros). Sin embargo, los materiales curriculares para las áreas lingüísticas no han sido el centro de atención hasta el momento. 
Por otra parte, en el artículo 6, sobre los criterios de homologación, ORDEN de 2 de septiembre de 2005 (Consejería de Educación, 2005b), por la que se establecen los criterios y normas sobre homologación de materiales curriculares para uso en los centros docentes, no se aprecia ningún criterio específico para poder homologar los libros de texto de cualquier lengua y, por ello, los miembros de este grupo de investigación observamos una laguna pedagógica considerable relativa a la adecuación de los libros de texto utilizados en los centros educativos a los contenidos del MCERL.

Por tanto, a tenor de lo anterior, estimamos necesario hacer un estudio que compruebe el grado de adecuación de los libros de texto de lenguas extranjeras utilizados en las aulas de nuestros centros con los niveles de competencia que marca el MCERL.

\section{Contexto}

Los libros de texto de inglés han sido objeto de investigación en numerosas ocasiones y por distintos motivos ${ }^{2}$ : por ejemplo, se ha investigado el grado de aceptación que tienen los libros de texto entre el alumnado (Palacios Martínez, 1994), o el uso que se hace de la L1 (español) en los libros de texto de inglés empleados en nuestro sistema educativo (Martín Martín, 1996). También se han abordado las cuestiones de género en los textos e ilustraciones (Cerezal, 1999; Stanley, 2001) de los materiales de enseñanza, o los elementos que tienen que ver con la representación de una sociedad intercultural en las imágenes y personajes incluidos en los mismos (Cerezal, Bazzano, Kasfiki, Martínez \& Monteverde, 1999). Otros estudios han prestado especial atención a los contenidos generales de los libros de textos en el periodo de vigencia de la LOGSE (Roldán Tapia, 2003) con una propuesta de sugerencias sobre cómo deberían ser los libros de texto desde el punto de vista del profesor, e incluso se ha ofrecido una visión global sobre los manuales utilizados en una quincena de países (Roldán Tapia, 2004). Interesante trabajo en relación a éste es el de Jacobs \& Ball (1996) que estudia la cantidad y el tipo de actividades de grupo incluidas en los libros de texto, con considerables diferencias entre los materiales analizados: en algunos textos las actividades para realizar en grupo suponen el $0 \%$ de su contenido mientras que en otros, para el mismo nivel, éstas alcanzan el $62 \%$ de las propuestas en el mismo.

De otra parte, un número de publicaciones recientes ha incidido en la importancia del MCERL en la didáctica de lenguas en general: se ha señalado la importancia de los aspectos socioculturales en el aprendizaje de lenguas y cómo éstos tienen cabida en la competencia socio-cultural (Guillén Díaz, 2005), así como su importancia como instrumento para la evaluación del aprendizaje de lenguas extranjeras (Montes Mozo, 2005). Díaz-Corralejo (20022003), Morrow (2004), Roza González (2006a) y Roza González (2006b) han presentado los aspectos generales del MCERL, mientras que McKenna Gleeson (2005) ha descrito la experimentación llevada a cabo en la Escuela Oficial de Idiomas de Córdoba para adecuar sus programaciones a los contenidos del Marco. En un sentido parecido, Keddle (1994) apunta las líneas de actuación para adecuar la programación en los centros de enseñanza secundaria al MCERL. Heyworth (2004) se pregunta por qué es importante el Marco, comentando, entre otros aspectos, la interrelación que debe existir a partir de ahora entre los libros de texto y las escalas de descriptores, que es en realidad el objetivo de nuestro estudio.

\footnotetext{
${ }^{2}$ Véase el trabajo de Roldán Tapia (2005: 132-135) para una revisión más extensa de estos estudios.
} 


\section{InVESTIGACIÓN}

\subsection{Objetivos de la investigación}

En el orden del propio trabajo de investigación, los objetivos planteados son los que siguen a continuación:

(a) estudiar la adecuación de los contenidos de los libros de texto a los niveles de competencia descritos en el MCERL;

(b) diseñar un instrumento de investigación, que sea útil para nuestro estudio, y que pueda generalizarse a estudios posteriores;

(c) elaborar un conjunto de propuestas, que puedan ir dirigidas al profesorado, a la administración educativa, la Consejería de Educación en este caso particular y a los autores y empresas editoras de los libros de texto.

\subsection{Diseño}

Tomando como referencia los cuatro parámetros descritos por Seliger \& Shohamy (1989: 24-40) para caracterizar un modelo de investigación:

Parámetro 1: Enfoque sintético (enfocado al todo) o analítico (enfocado a las partes).

Parámetro 2: Objetivos heurísticos (descriptivo) o deductivos (analiza hipótesis).

Parámetro 3: Grado de manipulación y control (alto o bajo) del contexto de investigación.

Parámetro 4: Modo de obtener los datos (poco estructurado o muy estructurado) y tipo de datos (cualitativos o cuantitativos).

La investigación que se presenta a continuación sobre el grado de adecuación de los libros de texto al MCERL, tendrá las siguientes variables:

P1

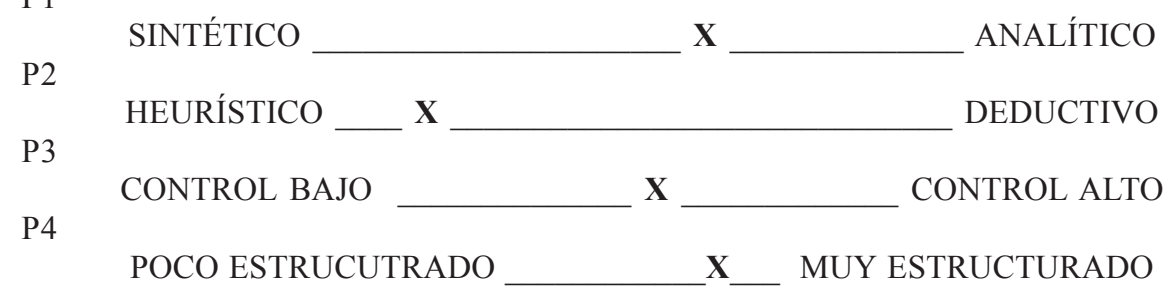

\subsection{Procedimiento y útiles de la investigación}

En la primera parte de la investigación, vamos a realizar un sondeo inicial para conocer cuáles son los libros de texto utilizados en $1^{\circ}$ de ESO en los centros de la provincia de Córdoba. Esto lo haremos a través del correo electrónico con mensajes dirigidos a los propios centros, para que los responda la Dirección o el propio Departamento de Inglés. Entendemos que por el amplio número de centros (174), los resultados se pueden extrapolar a otros ámbitos mayores. 
Una vez conocidos los textos que se usan, pasamos a hacer una selección de los más representativos por dos razones fundamentales: (a) las diferencias cuantitativas de uso son considerables entre los tres primeros, por ejemplo, y el resto, y (b) además, el tipo de observación de cada texto es muy exhaustivo, consumiendo una gran cantidad de tiempo y esfuerzo investigador. Se opta por convertir a los tres textos más empleados -New Thumbs Up, Exchange y United- en la base del estudio.

La segunda fase consistirá en una observación sistemática de todos y cada uno de los elementos que se incluyan en el registro de observación. Los elementos contenidos en este registro de observación se corresponderán con las escalas de descriptores que el MCERL utiliza para cada destreza lingüística en los niveles de competencia A1y A2.

Optamos por aunar las escalas de descriptores, siguiendo el modelo clásico de las cuatro destrezas: comprensión lectora y comprensión oral, producción escrita y producción oral. Intuimos que los niveles del MCERL que se corresponden con la enseñanza/aprendizaje de la lengua inglesa en $1^{\circ}$ de la ESO son el A1 y el A2, y es por ello que sólo los descriptores correspondientes a estos dos niveles de competencia son los que se van a incluir en el registro de observación. Quizás esta decisión pueda generar algún desconcierto si tenemos en cuenta los objetivos propuestos en los decretos que establecen las enseñanzas mínimas para la Primaria (RD 1531/2006) y Secundaria (RD 1631/2006), en lo que respecta a la lengua extranjera, pero la decisión se adopta por los siguientes motivos: (i) la experiencia acumulada a lo largo de los años nos dice que los contenidos presentados en los libros de texto de $1^{\circ}$ de la ESO tienen un carácter repetitivo de lo aprendido en la Primaria, y (iii) resulta sensato pensar que la superación de un nivel educativo no tenga una correspondencia unívoca con un nivel concreto del MCERL y que este nivel de competencia sea observable tanto en los materiales educativos utilizados en el final de una etapa educativa como en los empleados en el comienzo de la siguiente.

La fiabilidad de la investigación queda garantizada tanto en el procedimiento como en la obtención de los resultados. El registro de observación pasará un primer estudio de campo, exploratorio y no computable para la investigación. Este estudio de campo determinará su adecuación al objetivo que se persigue, servirá de entrenamiento para los observadores y permitirá hacer las modificaciones necesarias, si hubiere lugar a ello, para su utilización definitiva.

El registro de observación sistemática de esta investigación será aplicado por dos observadores, para cada libro de texto, para eliminar cualquier atisbo de subjetividad. En este sentido, calcularemos el índice de concordancia entre observadores, intentando que sea superior al 0.70 ó 0.80, tal y como indican los manuales de investigación educativa (Brown 1988:98 y Seliger \& Shohamy 1989:187), para que garantice la fiabilidad de los datos obtenidos.

\subsection{Bases del estudio}

Tomando como fuente de información a la dirección de los centros y a los datos complementados por las editoriales, los 174 libros utilizados en los IES, centros concertados y colegios de Primaria donde se imparte el $1^{\circ}$ ciclo de la ESO en la provincia de Córdoba son los que se presentan cuantificados a continuación: 
Tabla 1. Libros de texto de $1^{\circ}$ de ESO. Provincia de Córdoba

\begin{tabular}{|c|c|c|c|}
\hline Editorial & Libro de texto utilizado & Núm. de centros & Porcentaje \\
\hline OXFORD & THUMBS UP! NEW EDITION 1 & 41,00 & $24 \%$ \\
\hline OXFORD & EXCHANGE 1 & 20,00 & $11 \%$ \\
\hline MACMILLAN & UNITED 1 & 17,00 & $10 \%$ \\
\hline MACMILLAN & MACMILLAN SEC COURSE 1 & 13,00 & $7 \%$ \\
\hline BURLINGTON & CHANGES 1 & 11,00 & $6 \%$ \\
\hline LONGMAN & ENGLISH ZONE 1 & 9,00 & $5 \%$ \\
\hline SANTILLANA & DIFFERENT NEW EDITION 1 & 8,00 & $5 \%$ \\
\hline OXFORD & SPOTLIGHT 1 & 8,00 & $5 \%$ \\
\hline BURLINGTON & ENGLISH FOR YOU! 1 & 7,00 & $4 \%$ \\
\hline MACMILLAN & IMAGINE ENGLISH 1 & 7,00 & $4 \%$ \\
\hline BURLINGTON & LINKS 1 & 5,00 & $19 \%$ \\
\hline BURLINGTON & CHALLENGE FOR ESO 1 & 4,00 & \\
\hline SANTILLANA & CAN DO 1 & 4,00 & \\
\hline BURLINGTON & DISCOVER NEW EDITION 1 & 3,00 & \\
\hline S.M. & TEAM UP 1 & 3,00 & \\
\hline MACMILLAN & COSMOS 1 & 2,00 & \\
\hline OXFORD & ENGLISH ALIVE! 1 & 2,00 & \\
\hline OXFORD & OPEN DOORS PLUS 1 & 2,00 & \\
\hline MACMILLAN & SKILFUL 1 & 1,00 & \\
\hline LONGMAN & IN FOCUS 1 & 1,00 & \\
\hline SANTILLANA & YOUR TURN NEXT 1 & 1,00 & \\
\hline LONGMAN & WHATS UP? 1 & 1,00 & \\
\hline BURLINGTON & TOP CLASS NEW EDITION 1 & 1,00 & \\
\hline CAMBRIDGE & NEW WAYS TO GO 1 & 1,00 & \\
\hline OXFORD & HIGH SCORE 1 & 1,00 & \\
\hline ANAYA & MOVE 1 & 1,00 & \\
\hline \multirow[t]{2}{*}{ OXFORD } & OPEN DOORS 1 & 1,00 & \\
\hline & TOTAL & 174 & \\
\hline
\end{tabular}




\subsection{El registro de observación}

Dividido en cuatro apartados como se ha señalado anteriormente (comprensión lectora y oral, y producción escrita y oral), se elabora a partir de los descriptores que se presentan en el MCERL para los niveles A1 y A2. Hemos mantenido el registro en inglés para ser consecuentes con la lengua extranjera en la que se escriben los materiales propios de este estudio.

Por cuestiones obvias de espacio se presentan conjuntamente con los resultados de su aplicación.

\section{Resultados}

Por su extensión, se presentan al final del texto de este artículo, como documento anexo. Los descriptores que han sido observados en los textos, aparecen con un tick (P), quedando a disposición de los interesados los números de página y ejercicio en los que se observa la presencia de determinado descriptor en cada libro de texto.

\section{ANÁlisis y disCuSión de los RESUltados}

\subsection{Comprensión oral}

El primer dato destacable es el escaso número de descriptores observados para esta destreza lingüística, si bien el formato libro se ajusta difícilmente a algunos de los bloques de descriptores que conforman esta sección: así, los descriptores relativos a la comprensión de un interlocutor hablante nativo de la lengua y la comprensión de mensajes de televisión y vídeo no reflejan ninguna anotación.

El escaso número de descriptores también se ve incrementado por la carencia de los mismos para el nivel A1, de acuerdo con el MCERL, para la comprensión de una conversación entre hablantes nativos y la comprensión de mensajes grabados de audio.

Se echa en falta, quizás, más material de tipo auténtico en los textos a este nivel, de acuerdo con el número de descriptores observados, por ejemplo, para la comprensión de anuncios e instrucciones.

En términos generales, el libro de texto Exchange es el que menos descriptores recoge pero también es cierto que contiene una gran cantidad de actividades etiquetadas como Listening, en las que el alumno debe prestar atención a aspectos formales de la lengua y no al contenido de los mensajes que se transmiten.

Tal y como intuíamos en la introducción de nuestro proyecto, el volumen de descriptores correspondientes al nivel A1 es superior al de descriptores de A2, al menos siempre que existan para ambos niveles en cada uno de los bloques; compárese, por ejemplo, el bloque de conversación entre hablantes nativos y el de comprensión general.

\subsection{Comprensión lectora}

El número total de descriptores observados positivamente es superior al de la comprensión oral, pero también es evidente que el formato libro se ajusta mejor a este tipo de destreza 
lingüística. Además, no existe ningún bloque de descriptores carente de éstos para el nivel A1, como sí ocurre para la comprensión oral. En cualquier caso, es el libro de texto Exchange el que presenta un menor número de éstos en la suma de ambos observadores.

Comparativamente, los descriptores del nivel A1 ocurren con mucha mayor frecuencia que los descriptores del nivel A2, confirmando lo dicho con anterioridad en la comprensión oral.

En los tres libros, se percibe, al igual que para la comprensión oral, una deficiencia en el número de materiales auténticos utilizados para estas actividades de comprensión: así, por ejemplo, echamos en falta anuncios, prospectos, algún menú, carteles varios o instrucciones de algún objeto o electrodoméstico.

\subsection{Producción oral}

A pesar de ser la destreza para la que se contempla mayor número posible de posibles descriptores, los registrados por los observadores son menores en proporción al del resto de las otras tres destrezas. En el mejor de los casos, en el Exchange, se observan casi el $40 \%$ de los descriptores posibles, mientras que en el peor de los posibles, New Thumbs Up, se queda en el $26 \%$. Por tanto, el primer dato destacable es la desigualdad entre los tres textos, al menos en lo que respecta al formato libro, que ha sido el centro de nuestro estudio, y no otro material complementario de la misma serie. En esta línea, nos ha sorprendido que United incluya unos iconos específicos para indicar que la actividad en cuestión es de producción oral, mientras que no lo hace para las actividades de comprensión y producción escrita. Sin embargo, también incluye un icono para la comprensión oral.

En lo que respecta a los niveles de competencia, los descriptores para este curso de $1^{\circ}$ de ESO siguen estando repartidos entre el A1 y el A2, agudizándose para esta destreza la carencia de los mismos para el nivel más bajo, por ejemplo en los bloques de Public announcements o en Planning.

El otro dato importante es el número considerable de descriptores que no son observados en ninguna de las actividades de los tres libros de texto y que tienen que ver fundamentalmente con el proceso de interacción, más que con el contenido propio de la interacción; así, por ejemplo, no se registra ningún descriptor de categorías como Compensating, Identifying clues and inferring o Cooperating.

El mayor número de descriptores registrados tienen que ver con dos bloques bien característicos: aquél en el que los aprendices dan información sobre sí mismos y su entorno más cercano (Describing experience) y aquél otro en el que intercambian información de manera controlada con otro interlocutor (Conversation e Information Exchange). Esta circunstancia se produce de modo muy similar en los tres libros analizados.

\subsection{Producción escrita}

El primer dato que se evidencia es la proporción más elevada de descriptores observados, si la comparamos, por ejemplo, con la producción oral, llegando a estar en torno al $70 \%$ de ellos en el United. Es cierto que el formato libro ha podido ayudar a que se produzca esta circunstancia, yendo en detrimento de la anterior. Aunque con no muy acentuadas diferencias, se observa claramente un mayor índice de descriptores en United, con el índice más bajo en New Thumbs Up. 
Con respecto a los niveles de competencia, tanto los correspondientes a A1 como a A2 están recogidos en las actividades de los tres textos; véase, en particular, el bloque de producción escrita global.

En relación a los descriptores concretos que son observados, se detecta una diferencia considerable entre aquéllos en los que el alumno debe producir un texto en el que proporciona información sobre sí mismo y su entorno más cercano (Creative writing) y aquéllos otros en los que tiene que producir un texto con una finalidad comunicativa interactiva, como son por ejemplo escribir una postal, una nota o rellenar un formulario. En este sentido, echamos en falta la inclusión de algún material auténtico (algún impreso o formulario) con el que el alumno se pudiese encontrar al utilizar la segunda lengua fuera del aula.

\subsection{Análisis conjunto}

Del análisis conjunto de las cuatro destrezas en los tres libros de texto, se observa una diferencia importante entre el número de descriptores observados en los textos Exchange y United, frente a los observados en New Thumbs Up, que es, sin embargo, el libro de más amplio uso en todos los centros de la provincia.

Exchange destaca por el porcentaje tan alto registrado para la producción oral, en comparación con los otros dos textos, mientras que presenta el más bajo de los tres para la comprensión oral.

United, en particular, presenta los porcentajes más altos de los tres en los descriptores correspondientes a las dos destrezas de comprensión, la escrita y la oral.

\begin{tabular}{|c|c|c|c|c|c|c|}
\hline & \multicolumn{5}{|c|}{ NÚMERO Y PORCENTAJE DE DESCRIPTORES } \\
\hline & & $\begin{array}{c}\text { COMP. } \\
\text { LECTORA }\end{array}$ & $\begin{array}{c}\text { PROD. } \\
\text { ESCRITA }\end{array}$ & $\begin{array}{l}\text { COMP. } \\
\text { ORAL }\end{array}$ & $\begin{array}{l}\text { PROD. } \\
\text { ORAL }\end{array}$ & TOTAL \\
\hline \multirow[t]{2}{*}{ NTU } & $\mathrm{OB}_{1}^{3}$ & $\begin{array}{c}9 \\
(60 \%)\end{array}$ & $\begin{array}{c}8 \\
(47,05 \%)\end{array}$ & $\begin{array}{c}5 \\
(33,33 \%)\end{array}$ & $\begin{array}{c}20 \\
(26,66 \%)\end{array}$ & $\begin{array}{c}42 \\
(34,42 \%)\end{array}$ \\
\hline & $\mathrm{OB} 2$ & $\begin{array}{c}8 \\
(53,33 \%)\end{array}$ & $\begin{array}{c}10 \\
(58,82 \%)\end{array}$ & $\begin{array}{c}5 \\
(33,33 \%)\end{array}$ & $\begin{array}{c}20 \\
(26,66 \%)\end{array}$ & $\begin{array}{c}43 \\
(35,24 \%)\end{array}$ \\
\hline
\end{tabular}

\begin{tabular}{|c|c|c|c|c|c|c|}
\cline { 3 - 7 } \multicolumn{2}{c|}{} & \multicolumn{5}{c|}{ NÚMERO Y PORCENTAJE DE DESCRIPTORES } \\
\cline { 3 - 8 } \multicolumn{2}{c|}{} & $\begin{array}{c}\text { COMP. } \\
\text { LECTORA }\end{array}$ & $\begin{array}{c}\text { PROD. } \\
\text { ESCRITA }\end{array}$ & $\begin{array}{c}\text { COMP. } \\
\text { ORAL }\end{array}$ & PROD. ORAL & TOTAL \\
\hline \multirow{2}{*}{ EX } & OB1 & $\mathbf{6}$ & $\mathbf{1 0}$ & $\mathbf{4}$ & $\mathbf{3 0}$ & $\mathbf{5 0}$ \\
& & $\mathbf{( 4 0 \% )}$ & $\mathbf{( 5 8 , 8 2 \% )}$ & $\mathbf{( 2 6 , 6 6 \% )}$ & $\mathbf{( 4 0 \% )}$ & $\mathbf{( 4 0 , 9 8 \% )}$ \\
\cline { 2 - 8 } & OB2 & $\mathbf{8}$ & $\mathbf{1 1}$ & $\mathbf{4}$ & $\mathbf{2 9}$ & $\mathbf{5 2}$ \\
& & $\mathbf{( 5 3 , 3 3 \% )}$ & $\mathbf{( 6 4 , 7 0 \% )}$ & $\mathbf{( 2 6 , 6 6 \% )}$ & $\mathbf{( 3 8 , 6 6 \% )}$ & $\mathbf{( 4 2 , 6 2 \% )}$ \\
\hline
\end{tabular}

\begin{tabular}{|l|c|c|c|c|c|c|}
\cline { 3 - 7 } \multicolumn{2}{c|}{} & \multicolumn{5}{c|}{ NÚMERO Y PORCENTAJE DE DESCRIPTORES } \\
\cline { 3 - 7 } \multicolumn{2}{c|}{} & $\begin{array}{c}\text { COMP. } \\
\text { LECTORA }\end{array}$ & $\begin{array}{c}\text { PROD. } \\
\text { ESCRITA }\end{array}$ & $\begin{array}{c}\text { COMP. } \\
\text { ORAL }\end{array}$ & $\begin{array}{c}\text { PROD. } \\
\text { ORAL }\end{array}$ & TOTAL \\
\hline \multirow{3}{*}{ UN } & OB1 & $\mathbf{1 0}$ & $\mathbf{1 1}$ & $\mathbf{7}$ & $\mathbf{2 5}$ & $\mathbf{5 3}$ \\
& & $\mathbf{( 6 6 , 6 6 \% )}$ & $\mathbf{( 6 4 , 7 0 \% )}$ & $\mathbf{( 4 6 , 6 6 \% )}$ & $\mathbf{( 3 3 , 3 3 \% )}$ & $\mathbf{( 4 3 , 4 4 \% )}$ \\
\cline { 2 - 7 } & OB2 & $\mathbf{9}$ & $\mathbf{1 2}$ & $\mathbf{6}$ & $\mathbf{2 5}$ & $\mathbf{5 2}$ \\
& & $\mathbf{( 6 0 \% )}$ & $\mathbf{( 7 0 , 5 8 \% )}$ & $\mathbf{( 4 0 \% )}$ & $\mathbf{( 3 3 , 3 3 \% )}$ & $\mathbf{( 4 2 , 6 2 \% )}$ \\
\hline
\end{tabular}

\footnotetext{
${ }^{3}$ En esta tabla $n^{\circ} 2$ y en las tablas del apéndice se utilizan las iniciales OB1 y OB2, que se corresponden con cada uno de los dos observadores que han aplicado el registro de observación.
} 


\subsection{Trabajo de observación.}

Los datos presentados anteriormente y las afirmaciones hechas al respecto de los mismos demandan un control de fiabilidad que demuestre el rigor científico con el que se ha llevado la investigación.

En este dato los porcentajes de fiabilidad entre observadores (inter-rater reliability o inter-observer agreement, en la bibliografía especializada) están en todas las ocasiones por encima de lo que la teoría sobre investigación propone y las propias investigaciones presentan.

Tabla 3. Datos de fiabilidad entre observadores.

\begin{tabular}{|l|c|c|}
\cline { 2 - 3 } \multicolumn{1}{c|}{} & Número de descriptores & Descriptores concretos \\
\hline Comprensión lectora & $100 \%$ & $84 \%$ \\
\hline Producción escrita & $87,87 \%$ & $90,32 \%$ \\
\hline Comprensión oral & $94,44 \%$ & $97,14 \%$ \\
\hline Producción oral & $98,66 \%$ & $83,89 \%$ \\
\hline
\end{tabular}

\begin{tabular}{|l|c|}
\cline { 2 - 2 } \multicolumn{1}{c|}{} & Número de descriptores \\
\hline New Thumbs Up & $97,67 \%$ \\
\hline Exchange & $96,15 \%$ \\
\hline United & $98,11 \%$ \\
\hline TOTAL 3 libros (NTU + EX + UN) & $98,63 \%$ \\
\hline
\end{tabular}

Los coeficientes de fiabilidad entre observadores se presentan en un margen entre 0,00 y 1,00 , entendiéndose que una cifra que se encuentre entre 0,70 y 0,80 ya asegura un grado de fiabilidad aceptable. En todos los casos, nuestra investigación presenta unos coeficientes muy altos, tanto en los descriptores codificados $(83,89 \%-97,14 \%)$ como en el número de los mismos, tanto en el cómputo individual (87,87\%-100\%) de cada uno de ellos como en el total de todas las destrezas $(96,15 \%-98,11 \%)$.

Si a modo de comparación tomamos los coeficientes registrados en otros trabajos de investigación publicados, observamos que los nuestros no están lejos de los obtenidos en éstos: por ejemplo, los obtenidos por Pica \& Doughty (1985:238) que son de 0,88 y 0,93 , para su estudio del input negociado en grupo. Otros coeficientes observados (Jacobs \& Ball 1996:103) son algo más altos, 0,95 y 1 , si bien hay que decir en su contra que la muestra con la que se había medido era muy pequeña: solamente veinte actividades en grupo, extraídas de libros de texto. Nunan (1992:14-5) ofrece un coeficiente de 0,95 en una interacción de clase entre un alumno suyo y él mismo, pero no menciona ni el número de categorías codificadas ni da otra información relativa a la investigación realizada. Roldán Tapia (1997:263) obtiene coeficientes que varían entre un 0,83 y un 0,91 en su observación del trabajo en grupo en la realización de proyectos, con un registro que contiene 42 items.

\section{Conclusión}

En cuanto al objetivo (a) de la investigación, constatamos que nuestra intuición inicial era cierta y que los contenidos de los libros de texto de $1^{\circ}$ de ESO mantienen de forma reiterada una buena parte de los descriptores del nivel A1, ya iniciado en la Primaria, e introducen de forma generalizada el nivel de competencia A2 del MCERL. En este sentido, se puede afirmar 
que la adecuación de los tres textos a los descriptores correspondientes de ambos niveles es desigual, con un $10 \%$ de diferencia en el nivel de adecuación entre el más elevado (United) y el menos (New Thumbs Up). La adecuación de los tres libros de texto a los descriptores correspondientes de las cuatro competencias es en todo los casos superior en las destrezas escritas -comprensión lectora y producción escrita- frente a las orales -comprensión y producción oral-.

Con respecto al objetivo (b) podemos concluir que el carácter universal de los descriptores contenidos en el MCERL hace posible que su utilización como items de un registro de observación, den a éste una validez completa, permitiendo su aplicabilidad en cualquier otro estudio.

Tal y como proponíamos en el diseño de la investigación, el apartado de propuestas va a estar dividido en tres bloques, cuyos contenidos serán las líneas de actuación que se proponen para el profesorado, la Consejería de Educación y las empresas editoriales.

Para el profesorado, pensamos que sería muy conveniente que:

(1) hiciesen un esfuerzo por conocer con cierta profundidad el Marco Común Europeo de Referencia para las Lenguas (MCERL);

(2) empleasen el MCERL como un instrumento útil para la elección de los libros de texto que van a emplear con el alumnado;

(3) utilizasen el MCERL como instrumento de análisis y evaluación de los materiales de enseñanza existentes en el mercado y los disponibles en formato electrónico en la red;

(4) familiarizasen al alumnado con el MCERL, de forma que el desarrollo del Portfolio Europeo de las Lenguas (PEL) fuese una tarea factible en cada centro escolar.

A la propia Consejería de Educación de la Junta de Andalucía, como administración educativa, se le proponen las siguientes actuaciones:

(1) que utilice el MCERL como uno más de los mecanismos que le permiten adoptar decisiones sobre la homologación de libros de texto y materiales curriculares de aprendizaje de lenguas primera y extranjeras;

(2) que elabore un estudio que le permita determinar cuáles son los niveles de competencia deseables a la conclusión de cada etapa educativa o curso académico en el aprendizaje de las distintas lenguas que se ofertan en el sistema escolar;

(3) que tome las medidas necesarias para actualizar, por ejemplo, los temarios para las pruebas de acceso a cuerpos docentes, incluyendo entre éstos el MCERL y el PEL;

(4) que adopte las medidas necesarias para conjugar el modelo de enseñanza y aprendizaje de las competencias con el conocimiento y la aplicación del MCERL;

(5) que convierta el conocimiento del MCERL, si aún no lo es, en una de sus prioridades formativas para el profesorado que imparte algunas de las lenguas del sistema (Lengua Española, lenguas extranjeras $-1^{\mathrm{a}}$ y/o $2^{\mathrm{a}}-$, y Español para Extranjeros).

Las empresas editoras de libros de texto podrían tener en consideración las siguientes propuestas:

(1) que elaboren sus materiales de enseñanza, entre los que el libro de texto es el elemento articulador de la cada serie de los mismos, atendiendo a los descriptores que el MCERL establece para los distintos niveles de competencia que en él se describen; 
(2) que utilicen una serie de códigos o indicaciones (colores, iconos, etc.) comunes, por ejemplo, a todos los materiales que elaboran, por medio de los que el profesorado y el alumnado pueda saber en qué niveles del MCERL se encuentra el material que está utilizando;

(3) que incluyan información relativa a la adecuación de sus materiales de enseñanza al MCERL, particularmente en sus Teacher's;

(4) que hagan un esfuerzo por incluir materiales auténticos, al menos en los niveles educativos analizados en este proyecto, pues se ha observado una deficiencia considerable de éstos entre las actividades que desarrollan la comprensión lectora y oral y la producción escrita;

(5) que utilicen los niveles del MCERL para confeccionar los materiales suplementarios que facilitan al profesorado para poder atender al alumnado que precisa de algún tipo de adaptación curricular;

\section{RefERencias Bibliográficas}

Brown, J. D. (1988). Understanding research in second language learning. New York: Cambridge University Press.

Cerezal, F. (1999). La transmisión de valores genéricos discriminatorios en libros de texto en inglés. Alcalá de Henares: Servicio de Publicaciones de la Universidad de Alcalá.

CerezaL, F.; Bazzano, W.; Kasfiki, C.; Martínez, P. \& Monteverdi, A. (1999). «An intercultural approach to MLT textbooks evaluation», en Cerezal, F.; Megías, M. \& Jones, J. (Eds.). Reflexivity and interculturality in modern language teaching and learning. Madrid: Talasa, 63-76.

Consejería de Educación (2005a). «Acuerdo de 22 de marzo de 2005, del Consejo de Gobierno, por el que se aprueba el Plan de Fomento del Plurilingüismo en Andalucía», en BOJA número 65 de 5 de abril de 2005.

Consejería de Educación (2005b). «Orden de 2 de septiembre de 2005, por la que se establecen los criterios y normas sobre homologación de materiales curriculares para uso en los Centros docentes de Andalucía», en BOJA número 193 de 3 de octubre de 2005

Council of Europe (2001a). Common European Framework of Reference for languages: learning, teaching, assessment. Cambridge: Cambridge University Press and the Council of Europe.

Council of Europe (2001b). Recommendation 1539 (2001). European Year of Languages. [http:/ /assembly.coe.int]

Díaz-Corralejo, J. (2002-2003). «Marco Común Europeo de Referencia en el aprendizaje de lenguas extranjeras», en Encuentro. Revista de investigación e innovación en la clase de idioma, 13-14: $32-43$.

Guillén Díaz, C. (2005). «Los aspectos socioculturales y el Marco Europeo Común de Referencia para las Lenguas, en C. Montes Mozo (ed.). La enseñanza de las lenguas extranjeras desde una perspectiva europea. Madrid: Ministerio de Educación y Ciencia, 9-31.

Heyworth, F. (2004). "Why the CEF is important», in Morrow, K. (ed.). Insights from the Common European Framework. Oxford: Oxford University Press, 12-21.

Jacobs, G. M. \& J. Ball (1996). «An investigation of the structure of group activities in ELT coursebooks», en ELT Journal, 50, 2: 99-107.

Keddle, J. (2004). «The CEF and the secondary school syllabus», in Morrow, K. (ed.). Insights from the Common European Framework. Oxford: Oxford University Press, 43-54. 
Martín Martín, J. M. (1996). «Sobre el español en los libros de texto en inglés», en Donaire, 7: 3541.

McKenna Gleeson, J. (2005). «The Common European Framework: one experience of syllabus adaptation», en TESOL-Spain Newsletter, 29, July: 4-8.

Ministerio de Educación y Ciencia (2006). «Real Decreto 1513/2006, de 7 de diciembre, por el que se establecen las enseñanzas mínimas de la Educación primaria». $\mathrm{BOE}, \mathrm{n}^{\circ} 293$, de 8 de diciembre de 2006.

Ministerio de Educación y Ciencia (2007). «Real Decreto 1631/2006, de 29 de diciembre, por el que se establecen las enseñanzas mínimas correspondientes a la Educación Secundaria Obligatoria». BOE $\mathrm{n}^{\circ} 5$, de 5 de enero de 2007.

Montes Mozo, C. (2005). «La evaluación de lenguas extranjeras según las iniciativas y propuestas del Marco Común Eurpopeo de Referencia», en C. Montes Mozo (ed.). La enseñanza de las lenguas extranjeras desde una perspectiva europea. Madrid: Ministerio de Educación y Ciencia, 159-180.

Morrow, K. (2004). «Background to the CEF», in Morrow, K. (ed.). Insights from the Common European Framework. Oxford: Oxford University Press, 3-11.

Nunan, D. (1992). Research methods in language learning. Cambridge: Cambridge University Press.

Palacios Martínez, I. M. (1994). La enseñanza del inglés en España a debate. Santiago de Compostela: Servicio de Publicaciones e Intercambio Científico de la Universidad.

Pica, T. \& C. Doughty (1985). «The role of group work in classroom second language acquisition», en Studies in Second Language Acquisition, 7, 2: 233-248.

Roldán Tapia, A. R. (1997). La integración de un "process syllabus» en un curso de secundaria: input, interacción, destrezas, L1 y L2 en la realización de «project work». Granada: Servicio de Publicaciones de la Universidad.

Roldán Tapia, A. R. (2003). «EFL textbooks in Spanish schools (1990-2002)», en Guadalbullón (Revista de Educación de la Universidad de Jaén), 11: 53-66.

Roldán Tapia, A. R. (2004). «Textbooks around the world», en The APPI Journal (Revista de la Asociación Portuguesa de Profesores de Inglés), 4, 1: 33-36.

Roldán Tapia, A. R. (2005). «De Arthur \& Mary a Jack y Priya: Evolución de los libros de texto», en Cerezal, F. (ed.). De la práctica a la teoría. Reflexiones sobre la enseñanza y el aprendizaje de inglés. Alcalá de Henares: Servicio de Publicaciones de la Universidad de Alcalá: 129-145.

Roza González, M. B. (2006a). «Un proceso histórico hacia la convergencia en el plurilingüismo: el Marco Común Europeo de Referencia de las Lenguas», en TESOL-Spain Newsletter, 29, spring: 9-12.

Roza González, M. B. (2006b). «Un proceso histórico hacia la convergencia en el plurilingüismo: el Marco Común Europeo de Referencia de las Lenguas (parte 2)», en TESOL-Spain Newsletter, 30, July: 9-14.

Seliger, H. W. \& Shohamy, E. (1989). Second language research methods. Oxford: Oxford University Press.

Stanley, K. (2001). «TESL-EJ Forum: Sexist langugae in ESL/EFL textbooks and materials», en TESL-EJ 5/1. [http://cwp60.berkeley.edu:16080/TESL-EJ/ej17/f1.html] 


\section{Apéndice}

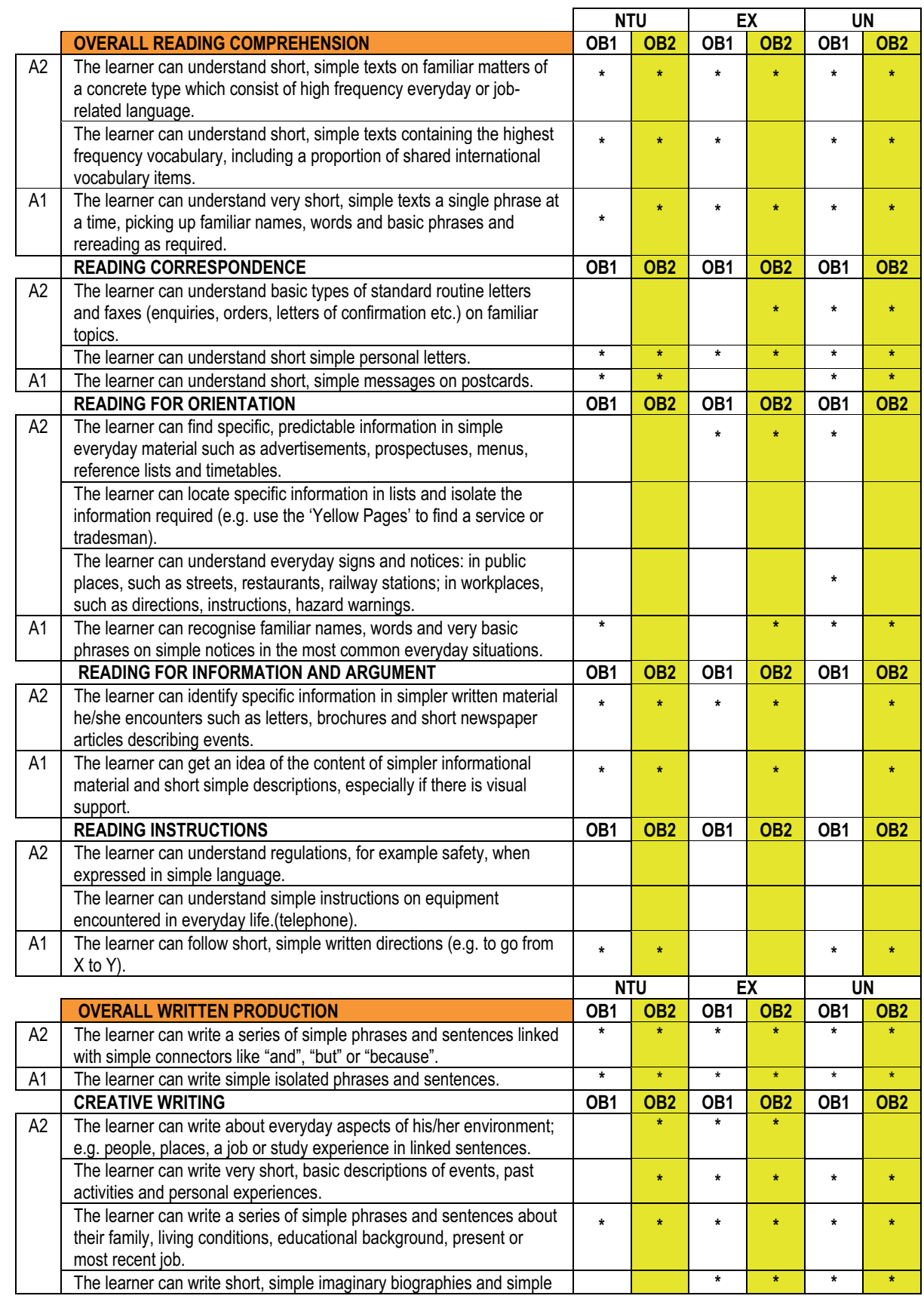




\begin{tabular}{|c|c|c|c|c|c|c|c|}
\hline & poems about people. & & & & & & \\
\hline \multirow[t]{2}{*}{$\mathrm{A} 1$} & $\begin{array}{l}\text { The learner can write simple phrases or sentences about themselves } \\
\text { and imaginary people, where they live and what they do. }\end{array}$ & * & * & * & * & * & * \\
\hline & OVERALL WRITTEN INTERACTION & OB1 & OB2 & OB1 & OB2 & OB1 & OB2 \\
\hline $\mathrm{A} 2$ & $\begin{array}{l}\text { The learner can write short, simple formulaic notes relating to matters } \\
\text { in areas of immediate need. }\end{array}$ & & & & * & * & \\
\hline \multirow[t]{2}{*}{$\mathrm{A} 1$} & The learner can ask for or pass on personal details in written form. & & & * & * & * & * \\
\hline & CORRESPONDENCE & OB1 & OB2 & OB1 & OB2 & OB1 & OB2 \\
\hline $\mathrm{A} 2$ & $\begin{array}{l}\text { The learner can write very simple personal letters expressing thanks } \\
\text { and apology. }\end{array}$ & & & & & & \\
\hline \multirow[t]{2}{*}{$\mathrm{A} 1$} & The learner can write a short simple postcard. & & & & & * & * \\
\hline & NOTES, MESSAGES AND FORMS & OB1 & OB2 & OB1 & OB2 & OB1 & OB2 \\
\hline \multirow[t]{2}{*}{$\mathrm{A} 2$} & $\begin{array}{l}\text { The learner can take a short, simple message provided he/she can } \\
\text { ask for repetition and reformulation. }\end{array}$ & & & & & & \\
\hline & $\begin{array}{l}\text { The learner can write short, simple notes and messages relating to } \\
\text { matters in areas of immediate need. }\end{array}$ & * & * & & & * & * \\
\hline \multirow[t]{2}{*}{$\mathrm{A} 1$} & $\begin{array}{l}\text { The learner can write numbers and dates, own name, nationality, } \\
\text { address, date of birth or arrival in the country, etc such as on a hotel } \\
\text { registration form. }\end{array}$ & & & & & & \\
\hline & PROCESSING TEXT & OB1 & OB2 & OB1 & OB2 & OB1 & OB2 \\
\hline \multirow[t]{2}{*}{$\mathrm{A} 2$} & $\begin{array}{l}\text { The learner can pick out and reproduce key words and phrases or } \\
\text { short sentences from a short text within the learner's limited } \\
\text { competence and experience. }\end{array}$ & * & * & * & * & * & * \\
\hline & $\begin{array}{l}\text { The learner can copy out short texts in printed or clearly handwritten } \\
\text { format. }\end{array}$ & * & * & * & * & & * \\
\hline $\mathrm{A} 1$ & $\begin{array}{l}\text { The learner can copy out single words and short texts presented in } \\
\text { standard printed format. }\end{array}$ & * & * & * & * & & * \\
\hline
\end{tabular}

\begin{tabular}{|c|c|c|c|c|c|c|c|}
\hline & \multirow[b]{2}{*}{ OVERALL LISTENING COMPREHENSION } & \multicolumn{2}{|c|}{ NTU } & \multicolumn{2}{|c|}{$\mathbf{E X}$} & \multicolumn{2}{|c|}{ UN } \\
\hline & & OB1 & OB2 & OB1 & OB2 & OB1 & OB2 \\
\hline \multirow[t]{2}{*}{$\mathrm{A} 2$} & $\begin{array}{l}\text { The learner can understand enough to be able to meet } \\
\text { needs of a concrete type provided speech is clearly and } \\
\text { slowly articulated. }\end{array}$ & & & & & * & \\
\hline & $\begin{array}{l}\text { The learner can understand phrases and expressions } \\
\text { related to areas of most immediate priority (e.g. very basic } \\
\text { personal and family information, shopping, local } \\
\text { geography, employment) provided speech is clearly and } \\
\text { slowly articulated. }\end{array}$ & $*$ & $*$ & $*$ & * & * & * \\
\hline \multirow[t]{2}{*}{ A1 } & $\begin{array}{l}\text { The learner can follow very slow and carefully articulated } \\
\text { speech, with long pauses for him/her to assimilate } \\
\text { meaning. }\end{array}$ & $*$ & * & $*$ & * & $*$ & * \\
\hline & $\begin{array}{l}\text { UNDERSTANDING CONVERSATION BETWEEN } \\
\text { NATIVE SPEAKERS }\end{array}$ & OB1 & OB2 & OB1 & OB2 & OB1 & OB2 \\
\hline \multirow[t]{3}{*}{$\mathrm{A} 2$} & $\begin{array}{l}\text { The learner can generally identify the topic of discussion } \\
\text { around her, when it is slowly and clearly. }\end{array}$ & $*$ & * & * & * & $*$ & $*$ \\
\hline & $\begin{array}{l}\text { UNDERSTANDING A NATIVE SPEAKER } \\
\text { INTERLOCUTOR [Ø] }\end{array}$ & OB1 & OB2 & OB1 & OB2 & OB1 & OB2 \\
\hline & $\begin{array}{l}\text { LISTENING TO ANNOUNCEMENTS AND } \\
\text { INSTRUCTIONS }\end{array}$ & OB1 & OB2 & OB1 & OB2 & OB1 & OB2 \\
\hline \multirow[t]{2}{*}{ A2 } & $\begin{array}{l}\text { The learner can catch the main point in short, clear, simple } \\
\text { messages and announcements. }\end{array}$ & & & & & & \\
\hline & $\begin{array}{l}\text { The learner can understand simple directions on how to } \\
\text { get from } \mathrm{X} \text { to } \mathrm{Y} \text {, by foot or public transport. }\end{array}$ & & & & & $*$ & * \\
\hline \multirow[t]{2}{*}{ A1 } & $\begin{array}{l}\text { The learner can understand instructions, carefully and } \\
\text { slowly addressed, and follow simple directions. }\end{array}$ & $*$ & $*$ & & & $*$ & $*$ \\
\hline & $\begin{array}{l}\text { LISTENING TO AUDIO MEDIA AND } \\
\text { RECORDINGS }\end{array}$ & OB1 & OB2 & OB1 & OB2 & OB1 & OB2 \\
\hline A2 & The learner can understand and extract the essential & $*$ & $*$ & $*$ & * & $*$ & $*$ \\
\hline
\end{tabular}




\begin{tabular}{|c|c|c|c|c|c|c|c|}
\hline & $\begin{array}{l}\text { information from short, recorded passages dealing with } \\
\text { predictable everyday matters which are delivered slowly } \\
\text { and clearly. }\end{array}$ & & & & & & \\
\hline & WATCHING TV AND FILM [Ø] & OB1 & OB2 & OB1 & OB2 & OB1 & OB2 \\
\hline & & \multicolumn{2}{|c|}{ NTU } & \multicolumn{2}{|c|}{ EX } & \multicolumn{2}{|c|}{$\mathbf{U N}$} \\
\hline & OVERALL ORAL PRODUCTION & OB1 & OB2 & OB1 & OB2 & OB1 & OB2 \\
\hline $\mathrm{A} 2$ & $\begin{array}{l}\text { The learner can give a simple description or presentation } \\
\text { of people, living or working conditions, daily routines, } \\
\text { likes/dislikes, etc. as a short series of simple phrases and } \\
\text { sentences linked into a list. }\end{array}$ & & & * & & * & \\
\hline \multirow[t]{2}{*}{ A1 } & $\begin{array}{l}\text { The learner can produce simple mainly isolated phrases } \\
\text { about people and places. }\end{array}$ & $*$ & * & $*$ & $*$ & $*$ & * \\
\hline & $\begin{array}{lll}\text { SUSTAINED } & \text { MONOLOGUE: } & \text { DESCRIBING } \\
\text { EXPERIENCE } & & \\
\end{array}$ & OB1 & OB2 & OB1 & OB2 & OB1 & OB2 \\
\hline \multirow[t]{8}{*}{ A2 } & $\begin{array}{l}\text { The learner can tell a story or describe something in a } \\
\text { simple list of points. }\end{array}$ & * & * & $*$ & * & * & * \\
\hline & $\begin{array}{l}\text { The learner can describe everyday aspects of his/her } \\
\text { environment e.g. people, places, a job or study. }\end{array}$ & $*$ & * & $*$ & * & * & $*$ \\
\hline & $\begin{array}{l}\text { The learner can give short, basic descriptions of events } \\
\text { and activities. }\end{array}$ & $*$ & $*$ & $*$ & $*$ & * & * \\
\hline & $\begin{array}{l}\text { The learner can describe plans and arrangements, routines, } \\
\text { past activities and personal experiences. }\end{array}$ & $*$ & * & $*$ & $*$ & * & * \\
\hline & $\begin{array}{l}\text { The learner can use simple descriptive language to make } \\
\text { brief statements about and compare objects and } \\
\text { possessions. }\end{array}$ & $*$ & * & $*$ & $*$ & $*$ & $*$ \\
\hline & $\begin{array}{l}\text { The learner can explain what he/she likes or dislikes about } \\
\text { something. }\end{array}$ & $*$ & * & $*$ & $*$ & $*$ & $*$ \\
\hline & $\begin{array}{l}\text { The learner can describe his/her family, living conditions, } \\
\text { educational background, present or most recent job. }\end{array}$ & $*$ & * & $*$ & * & * & * \\
\hline & $\begin{array}{l}\text { The learner can describe people, places and possessions in } \\
\text { simple terms. }\end{array}$ & $*$ & * & * & * & * & * \\
\hline \multirow[t]{2}{*}{ A1 } & $\begin{array}{l}\text { The learner can describe him/herself, what he/she does and } \\
\text { where he/she lives. }\end{array}$ & $*$ & * & * & * & * & * \\
\hline & PUBLIC ANNOUNCEMENTS & OB1 & OB2 & OB1 & OB2 & OB1 & OB2 \\
\hline \multirow[t]{2}{*}{ A2 } & $\begin{array}{l}\text { The learner can deliver very short, rehearsed } \\
\text { announcements of predictable, learnt content which are } \\
\text { intelligible to listeners who are prepared to concentrate. }\end{array}$ & & & & & * & * \\
\hline & ADDRESSING AUDIENCES & OB1 & OB2 & OB1 & OB2 & OB1 & OB2 \\
\hline \multirow[t]{2}{*}{$\mathrm{A} 2$} & $\begin{array}{l}\text { The learner can give a short, rehearsed, basic presentation } \\
\text { on a familiar subject. }\end{array}$ & & & & & $*$ & $*$ \\
\hline & $\begin{array}{l}\text { The learner can answer straightforward follow up } \\
\text { questions if he/she can ask for repetition and if some help } \\
\text { with the formulation of his/her reply is possible. }\end{array}$ & & & & & & \\
\hline \multirow[t]{2}{*}{ Al } & $\begin{array}{l}\text { The learner can read a very short, rehearsed statement - } \\
\text { e.g. to introduce a speaker, propose a toast. }\end{array}$ & $*$ & * & & & & * \\
\hline & OVERALL ORAL STRATEGIES: PLANNING & OB1 & OB2 & OB1 & OB2 & OB1 & OB2 \\
\hline \multirow[t]{4}{*}{$\mathrm{A} 2$} & $\begin{array}{l}\text { The learner can recall and rehearse an appropriate set of } \\
\text { phrases from his/her repertoire. }\end{array}$ & * & $*$ & $*$ & $*$ & & \\
\hline & COMPENSATING [Ø] & OB1 & OB2 & OB1 & OB2 & OB1 & OB2 \\
\hline & $\begin{array}{l}\text { RECEPTION STRATEGIES: IDENTIFYING CUES } \\
\text { AND INFERRING [Ø] }\end{array}$ & OB1 & OB2 & OB1 & OB2 & OB1 & OB2 \\
\hline & $\begin{array}{l}\text { INTERACTIVE ACTIVITIES AND STRATEGIES: } \\
\text { OVERALL SPOKEN INTERACTION }\end{array}$ & OB1 & OB2 & OB1 & OB2 & OB1 & OB2 \\
\hline \multirow[t]{3}{*}{ A2 } & $\begin{array}{l}\text { The learner can interact with reasonable ease in structured } \\
\text { situations and short conversations, provided the other } \\
\text { person helps if necessary. }\end{array}$ & & & & & * & \\
\hline & $\begin{array}{l}\text { The learner can manage simple, routine exchanges without } \\
\text { undue effort. }\end{array}$ & $*$ & $*$ & $*$ & $*$ & $*$ & * \\
\hline & The learner can ask and answer questions and exchange & * & * & $*$ & * & * & * \\
\hline
\end{tabular}




\begin{tabular}{|c|c|c|c|c|c|c|c|}
\hline & $\begin{array}{l}\text { ideas and information on familiar topics in predictable } \\
\text { everyday situations. }\end{array}$ & & & & & & \\
\hline & $\begin{array}{l}\text { The learner can communicate in simple and routine tasks } \\
\text { requiring a simple and direct exchange of information on } \\
\text { familiar and routine matters to do with work and free time. }\end{array}$ & $*$ & * & & & & * \\
\hline & $\begin{array}{l}\text { The learner can handle very short social exchanges but is } \\
\text { rarely able to understand enough to keep conversation } \\
\text { going of his/her own accord. }\end{array}$ & & & & & & \\
\hline A1 & $\begin{array}{l}\text { The learner can interact in a simple way but } \\
\text { communication is totally dependent on repetition at a } \\
\text { slower rate of speech, rephrasing and repair. }\end{array}$ & $*$ & $*$ & $*$ & $*$ & * & $*$ \\
\hline & $\begin{array}{l}\text { The learner can ask and answer simple questions, initiate } \\
\text { and respond to simple statements in areas of immediate } \\
\text { need or on very familiar topics. }\end{array}$ & $*$ & $*$ & $*$ & + & $*$ & $*$ \\
\hline & CONVERSATION & OB1 & OB2 & OB1 & OB2 & OB1 & OB2 \\
\hline $\mathrm{A} 2$ & $\begin{array}{l}\text { The learner can handle very short social exchanges but is } \\
\text { rarely able to understand enough to keep conversation } \\
\text { going of his/her own accord, though he/she can be made to } \\
\text { understand if the speaker will take the trouble. }\end{array}$ & * & & & & & \\
\hline & $\begin{array}{l}\text { The learner can use simple everyday polite forms of } \\
\text { greeting and address. }\end{array}$ & & & $*$ & & * & \\
\hline & $\begin{array}{l}\text { The learner can make and respond to invitations, } \\
\text { suggestions and apologies. }\end{array}$ & & & $*$ & & & * \\
\hline & The learner can say what he/she likes and dislikes. & $*$ & $*$ & $*$ & $*$ & & \\
\hline A1 & $\begin{array}{l}\text { The learner can make an introduction and use basic } \\
\text { greeting and leave-taking expressions }\end{array}$ & & & $*$ & $*$ & & \\
\hline & The learner can ask how people are and react to news. & & * & & $*$ & & \\
\hline & $\begin{array}{l}\text { The learner can understand everyday expressions aimed at } \\
\text { the satisfaction of simple needs of a concrete type, } \\
\text { delivered directly to him/her in clear, slow and repeated } \\
\text { speech by a sympathetic speaker. }\end{array}$ & * & & & & * & \\
\hline & INFORMAL DISCUSSION: with friends & OB1 & OB2 & OB1 & OB2 & OB1 & OB2 \\
\hline $\mathrm{A} 2$ & $\begin{array}{l}\text { The learner can identify the topic of discussion around } \\
\text { him/her when it is conducted slowly and clearly. }\end{array}$ & & & & & & \\
\hline & $\begin{array}{l}\text { The learner can discuss what to do in the evening, at the } \\
\text { weekend. }\end{array}$ & & & $*$ & $*$ & & \\
\hline & The learner can make and respond to suggestions. & * & * & $*$ & * & & \\
\hline & The learner can agree and disagree with others. & & & & & & \\
\hline & $\begin{array}{l}\text { The learner can discuss everyday issues in a simple way } \\
\text { when addressed clearly, slowly and directly. }\end{array}$ & & & & & & \\
\hline & $\begin{array}{l}\text { The learner can discuss what to do, where to go and make } \\
\text { arrangements to meet. }\end{array}$ & & & * & * & & \\
\hline & FORMAL DISCUSSIONS AND MEETINGS [Ø] & OB1 & OB2 & OB1 & OB2 & OB1 & OB2 \\
\hline & $\begin{array}{l}\text { GOAL-ORIENTED CO-OPERATION (e.g., discussing } \\
\text { a document, organising an event) [Ø] }\end{array}$ & OB1 & OB2 & OB1 & OB2 & OB1 & OB2 \\
\hline & $\begin{array}{l}\text { TRANSACTIONS TO OBTAIN GOODS } \\
\text { SERVICES }\end{array}$ & OB1 & OB2 & OB1 & OB2 & OB1 & OB2 \\
\hline A2 & $\begin{array}{l}\text { The learner can ask for and provide everyday goods and } \\
\text { services. }\end{array}$ & & & & & & \\
\hline & $\begin{array}{l}\text { The learner can get simple information about travel, use } \\
\text { public transport: buses, trains, and taxis, ask and give } \\
\text { directions, and buy tickets }\end{array}$ & & & & & & \\
\hline & $\begin{array}{l}\text { The learner can ask about things and make simple } \\
\text { transactions in shops, post offices or banks. }\end{array}$ & & & $*$ & $*$ & & \\
\hline & $\begin{array}{l}\text { The learner can give and receive information about } \\
\text { quantities, numbers, prices, etc. }\end{array}$ & & & * & $*$ & & \\
\hline & $\begin{array}{l}\text { The learner can make simple purchases by stating what is } \\
\text { wanted and asking the price. }\end{array}$ & & & $*$ & * & & \\
\hline & The learner can order a meal. & & & & & & \\
\hline
\end{tabular}




\begin{tabular}{|c|c|c|c|c|c|c|c|}
\hline \multirow[t]{3}{*}{ A1 } & $\begin{array}{l}\text { The learner can ask people for things and give people } \\
\text { things. }\end{array}$ & & & & & & \\
\hline & The learner can handle numbers, quantities, cost and time. & $*$ & $*$ & & & $*$ & * \\
\hline & INFORMATION EXCHANGE & OB1 & OB2 & OB1 & OB2 & OB1 & OB2 \\
\hline \multirow[t]{8}{*}{ A2 } & $\begin{array}{l}\text { The learner can ask and answer questions about habits and } \\
\text { routines. }\end{array}$ & & & & $*$ & $*$ & * \\
\hline & $\begin{array}{l}\text { The learner can ask and answer questions about pastimes } \\
\text { and past activities. }\end{array}$ & $*$ & * & $*$ & $*$ & * & * \\
\hline & $\begin{array}{l}\text { The learner can give and follow simple directions and } \\
\text { instructions, e.g. explain how to get somewhere. }\end{array}$ & & & & & $*$ & * \\
\hline & $\begin{array}{l}\text { The learner can communicate in tasks requiring a simple } \\
\text { and direct exchange of information. }\end{array}$ & & & & & & * \\
\hline & $\begin{array}{l}\text { The learner can exchange limited information on familiar } \\
\text { and routine operational matters }\end{array}$ & * & * & & * & $*$ & * \\
\hline & $\begin{array}{l}\text { The learner can ask and answer questions about what they } \\
\text { do at work and in free time. }\end{array}$ & & * & & $*$ & $*$ & * \\
\hline & $\begin{array}{l}\text { The learner can ask for and give directions referring to a } \\
\text { map or plan }\end{array}$ & & & & $*$ & * & * \\
\hline & The learner can ask for and provide personal information. & $*$ & * & $*$ & $*$ & $*$ & * \\
\hline \multirow[t]{4}{*}{$\mathrm{A} 1$} & $\begin{array}{l}\text { The learner can ask and answer simple questions, initiate } \\
\text { and respond to simple statements in areas of immediate } \\
\text { need or on very familiar topics. }\end{array}$ & $*$ & * & $*$ & $*$ & $*$ & * \\
\hline & $\begin{array}{l}\text { The learner can ask and answer questions about } \\
\text { themselves and other people, where they live, people they } \\
\text { know, things they have. }\end{array}$ & $*$ & * & & $*$ & $*$ & * \\
\hline & $\begin{array}{l}\text { The learner can indicate time by such phrases as next } \\
\text { week, last Friday, in November, three o'clock. }\end{array}$ & $*$ & $*$ & * & & $*$ & $*$ \\
\hline & INTERVIEWING AND BEING INTERVIEWED & OB1 & OB2 & OB1 & OB2 & OB1 & OB2 \\
\hline \multirow[t]{2}{*}{$\mathrm{A} 2$} & $\begin{array}{l}\text { The learner can make her understood in an interview and } \\
\text { communicate ideas and information on familiar topics, } \\
\text { provided she can ask for clarification occasionally, and is } \\
\text { given some help to express what he/she wants to. }\end{array}$ & & & & & & \\
\hline & $\begin{array}{l}\text { The learner can answer simple questions and respond to } \\
\text { simple statements in an interview. }\end{array}$ & $*$ & * & & & $*$ & \\
\hline \multirow[t]{2}{*}{ A1 } & $\begin{array}{l}\text { The learner can reply in an interview to simple direct } \\
\text { questions spoken very slowly and clearly in direct non- } \\
\text { idiomatic speech about personal details. }\end{array}$ & $*$ & * & & & $*$ & \\
\hline & TAKING THE FLOOR: TURNTAKING. & OB1 & OB2 & OB1 & OB2 & OB1 & OB2 \\
\hline \multirow[t]{5}{*}{ A2 } & $\begin{array}{l}\text { The learner can use simple techniques to start, maintain, or } \\
\text { end a short conversation. }\end{array}$ & & & * & & & \\
\hline & $\begin{array}{l}\text { The learner can initiate, maintain and close simple, face- } \\
\text { to-face conversation. }\end{array}$ & & & $*$ & * & & \\
\hline & The learner can ask for attention. & & & & & & \\
\hline & COOPERATING [Ø] & OB1 & OB2 & OB1 & OB2 & OB1 & OB2 \\
\hline & ASKING FOR CLARIFICATION & OB1 & OB2 & OB1 & OB2 & OB1 & OB2 \\
\hline \multirow[t]{3}{*}{ A2 } & $\begin{array}{l}\text { The learner can ask very simply for repetition when he/she } \\
\text { does not understand. }\end{array}$ & & & & & & \\
\hline & $\begin{array}{l}\text { The learner can ask for clarification about key words or } \\
\text { phrases not understood using stock phrases. }\end{array}$ & & & * & * & & \\
\hline & The learner can say he/she didn't follow. & & & & & & \\
\hline
\end{tabular}

